\title{
WINDING NUMBER AND THE NUMBER OF REAL ZEROS OF A FUNCTION
}

\author{
LIEVEN SMITS AND WILLEM KUYK
}

(Communicated by R. Daniel Mauldin)

\begin{abstract}
The theorem in this paper shows that the number of real simple zeros of a function of the form $f(x)=q(x)+a x+b, x \in \mathbb{R}$, for not too wild $q(x)$ can be obtained counting the winding number of a closed plane curve about the point $(a, b)$.
\end{abstract}

\section{INTRODUCTION AND PRELIMINARIES}

Let $q$ be a twice continuously differentiable function from $\mathbb{R}$ to $\mathbb{R}$ and put

$$
f(x)=q(x)+a x+b, \quad x \in \mathbb{R},
$$

where $a$ and $b$ are real constants. In this paper we shall first give a parametrization of the set of ordered pairs $(a, b)$ such that $f$ has a nonsimple zero, and then construct a geometric picture of the dependence of the number of zeros of $f$ on $a$ and $b$, provided that they are simple. We then determine the number of real zeros of $f$ for a large class of functions $q$ that includes all polynomial functions of degree greater than one.

Note that $f$ has a nonsimple zero at $x \in \mathbb{R}$ if and only if

$$
\begin{aligned}
& \left\{\begin{array}{l}
f(x)=0 \\
f^{\prime}(x)=0
\end{array}\right. \\
& \Longleftrightarrow\left\{\begin{array}{l}
a=-q^{\prime}(x) \\
b=x q^{\prime}(x)-q(x) .
\end{array}\right.
\end{aligned}
$$

This parametrizes what we shall call the catastrophe curve cat $(q)$ of $q$ in the $(a, b)$-plane. Note that curves of this type with $q(x)$ a polynomial occur in a natural way in the geometry of catastrophe types with one state parameter [1].

Lemma 1. $\operatorname{cat}(q)$ is isotangent, i.e., the slope at a parameter point $x$ is equal to $-x$.

Proof. One calculates

$$
\begin{gathered}
a^{\prime}(x)=-q^{\prime \prime}(x), \\
b^{\prime}(x)=q^{\prime}(x)+x q^{\prime \prime}(x)-q^{\prime}(x)=-x a^{\prime}(x) .
\end{gathered}
$$

Received by the editors June 27, 1990.

1980 Mathematics Subject Classification (1985 Revision). Primary 26C10; Secondary 12D10.

Both authors acknowledge the support of the Belgian National Science Foundation NFWO. 
From now on we suppose that the function $q$ satisfies the following axioms 1 and 3 , and either axiom $2 \mathrm{a}$ or $2 \mathrm{~b}$ :

(1) $\lim _{x \rightarrow+\infty} q^{\prime}(x)=+\infty$,

(2a) $\lim _{x \rightarrow-\infty} q^{\prime}(x)=+\infty$ (called the "odd case"),

(2b) $\lim _{x \rightarrow-\infty} q^{\prime}(x)=-\infty$ (called the "even case"),

(3) $q^{\prime \prime}$ has all its zeros in a compact interval.

Remark. Note that these axioms are satisfied if $q$ is a monic polynomial of degree at least two, and in that case the terms odd/even refer precisely to the degree of the polynomial.

Lemma 2. The catastrophe curve cat $(q)$ tends to infinity in specific quadrants, more precisely:

$$
\begin{aligned}
-\lim _{x \rightarrow+\infty} a(x) & =+\lim _{x \rightarrow+\infty} b(x)=+\infty, \\
\lim _{x \rightarrow-\infty} a(x) & =\lim _{x \rightarrow-\infty} b(x)= \pm \infty \quad \text { (even/odd). }
\end{aligned}
$$

Proof. The statements for $a( \pm \infty)$ follow from $a(x)=-q^{\prime}(x)$ and from the axioms. To compute $b(+\infty)$, choose $\alpha>1$ such that $a^{\prime}(x)<0$ for $x \geq \alpha$. Next choose $\beta>\alpha$ such that $a(x)<a(\alpha)+b(\alpha)$ for $x \geq \beta$, which is possible on account of $a(+\infty)=-\infty$. By Cauchy's mean value theorem, for all $x>\beta$, there exists a $\gamma \in(\beta, x)$ such that

$$
a^{\prime}(\gamma)(b(x)-b(\beta))=b^{\prime}(\gamma)(a(x)-a(\beta)) .
$$

As $\operatorname{cat}(q)$ is isotangent, and since $a(x)-a(\beta)<0$ there exists for all $x>\beta$ a $\gamma>\beta$ such that

$$
\begin{aligned}
b(x) & =b(\beta)-\gamma(a(x)-a(\beta)) \\
& \geq b(\beta)-\beta(a(x)-a(\beta)) \rightarrow+\infty \quad \text { as } x \rightarrow+\infty .
\end{aligned}
$$

Analogous proofs work for $b(-\infty)$ in the even and odd cases.

Lemma 3. There exists an $R_{0}>0$ such that, if $R \geq R_{0}$, the circle $C(0, R)$ with radius $R$ centered at the origin has two points in common with the image of $\operatorname{cat}(q)$.

Proof. We shall prove that, ultimately, the branches of $\operatorname{cat}(q)$ go to infinity in a monotonic way. Consider the square norm

$$
s(x)=a(x)^{2}+b(x)^{2} .
$$

First note that $s \geq a^{2}=\left(q^{\prime}\right)^{2} \rightarrow+\infty$. If follows that, for large radius $R$, there are at least two values of the parameter $x$ where $\operatorname{cat}(q)$ intersects $C(0, R)$.

Next, its derivative is

$$
s^{\prime}=2 a a^{\prime}+2 b b^{\prime}=2 q^{\prime \prime}\left(q^{\prime}+x^{2} q^{\prime}-x q\right) .
$$

By the axioms, $q^{\prime}$ has constant sign outside a compact interval. But so does $x^{2} q^{\prime}-x q$, and the sign is the same:

$$
x q^{\prime}(x)-q(x)=q(0)+\int_{t=0}^{x}\left(q^{\prime}(x)-q^{\prime}(t)\right) d t \rightarrow \pm \infty,
$$

the sign depending on the cases odd/even and $x \rightarrow \pm \infty$. In fact, one verifies that $s^{\prime}$ is negative on a left half line, and positive on a right half line (with possibly several sign changes in between). 
It follows that large circles intersect $\operatorname{cat}(q)$ at exactly two values of the parameter. By Lemma 2, these values yield distinct points.

\section{NOTATIONS AND THE CONCEPT OF LIMITING WINDING NUMBER}

In the following we shall identify the topological one-torus, i.e. the interval $[0,2 \pi]$ with the end points identified, with the collection of all half lines in the plane with a fixed starting point. An anticlockwise motion in this torus (with respect to a fixed coordinate system, say $X Y$ ) is a differentiable nonwinding (i.e. injective) path which encounters the unit vectors on the $X$ and $Y$ axes in the order $\left(e_{X}, e_{Y},-e_{X},-e_{Y}\right)$. A clockwise motion has the opposite orientation. An interval $[a, b]$ on the torus, for $a \neq b$, is chosen such that running through it is an anticlockwise motion. With this convention, we choose the range of the Arctg function to be $(-\pi / 2, \pi / 2)=(3 \pi / 2, \pi / 2)$.

As before, let $C(0, R)$ be the circle in the $(a, b)$-plane with radius $R>0$ centered at the origin. For any half line $L$ in the plane, we denote by $\widetilde{L}$ the complementary half line with the same support, i.e., $L \cup \widetilde{L}$ is a line.

By an increasing (resp. decreasing) zero of a real function we mean a value in its domain $\mathbb{R}$ which is mapped to 0 , together with a neighborhood of that value on which the function is strictly increasing (resp. decreasing). Classically, a simple zero of a continuously differentiable function is necessarily either an increasing or a decreasing one.

Definition. Let $q, c, d$ and $R_{0}$ be as in the previous lemmas. The limiting winding number $W(q, c, d)$ for a point $(c, d)$ in the plane not belonging to the image of cat $(q)$, is the winding number about $(c, d)$ of a closed oriented curve $\mathbf{S}$ in $\mathbb{R}^{2} \backslash\{(c, d)\}$, defined by the following construction.

Take an arbitrary $R>\max \left(R_{0}, \sqrt{c^{2}+d^{2}}\right)$ and let $x_{0}<x_{1}$ be the parameter values of $\operatorname{cat}(q)$ producing the intersection points of Lemma 3 . The curve $\mathbf{S}$ is constructed by glueing together the following two pieces:

- $\operatorname{cat}(q)$ from $x_{0}$ to $x_{1}$,

- a segment of $C(0, R)$, oriented anticlockwise from $\left(a\left(x_{1}\right), b\left(x_{1}\right)\right)$ to $\left(a\left(x_{0}\right), b\left(x_{0}\right)\right)$.

Note that this definition is independent of the particular choice of $R>$ $\max \left(R_{0}, \sqrt{c^{2}+d^{2}}\right)$, since changing $R$ is a homotopy of curves in $\mathbb{R}^{2} \backslash\{(c, d)\}$ (one may construct the homotopy explicitly using a reparametrization of some patches by the parameter $s$ of Lemma 3 ).

\section{THE WINDING NUMBER THEOREM}

Theorem. In the odd case, the number of real zeros of

$$
f(x)=q(x)+a x+b
$$

for $(a, b) \notin \operatorname{cat}(q)$, is equal to $1+2 W(q, a, b)$. In the even case, it is $2 W(q, a, b)$.

Remark. The proof is rather technical. Figure 1 shows separately what is going on in the odd and even cases. The theorem concerns the closed bold curve that winds around the point $(a, b)$. The drawings of these curves are generic, in 
the sense that no elements special to any particular case of $\operatorname{cat}(q)$ are used. For the proof of the theorem it is not necessary to draw in each case that part of $\operatorname{cat}(q)$ that lies inside the circle and that contains the cusps of cat $(q)$. Whence the incomplete drawings of the bold curves. The reader may wish to convince himself by drawing the curves $\operatorname{cat}(q)$ for the special cases

$$
q(x)=x^{3} \quad \text { (odd case), } \quad q(x)=x^{4}-2 x^{2} \quad \text { (even case). }
$$

Proof. Since $(a, b) \notin \operatorname{cat}(q)$, all zeros are simple. We shall prove that the number of decreasing zeros of $f(x)$ is equal to $W(q, a, b)$. Since, in the odd case,

$$
\lim _{x \rightarrow \pm \infty} q^{\prime}(x)=+\infty
$$

we have

$$
\lim _{x \rightarrow+\infty} f(x)=-\lim _{x \rightarrow-\infty} f(x)=+\infty
$$

(with obvious analogues in the even case), and the theorem will be established.

Denoting a point $(a, b)$ on $\operatorname{cat}(q)$ by capital letters, observe first that, for all $x \in \mathbb{R}$, the tangent to cat $(q)$ at $(A(x), B(x))$ has the following equation in the $(a, b)$-plane

$$
T_{x} \longleftrightarrow x \cdot a+b+q(x)=0 .
$$

At the point $(a, b)=(A(x), B(x))$ of this line, we have

$$
a+q^{\prime}(x)=0 \text {. }
$$

Furthermore, this line $T_{x}$ has slope $-x \neq \infty$, so it cannot be parallel to the axis $b=0$. We conclude that the quantity $\partial f / \partial x=a+q^{\prime}(x)$, for the same fixed $x$, is negative for $(a, b)$ on the open half line $S_{x}$ of $T_{x}$ which starts at $(A(x), B(x))$ and points into the negative $a$ direction. Thus the real number $x$ is a decreasing zero of $q(x)+a x+b$ if and only if $(a, b)$ lies on the half line $S_{x}$.

The proof of the theorem proceeds in constructing a new closed curve $P=$ $\left(p_{1}(\theta), p_{2}(\theta)\right)$ in $\mathbb{R}^{2} \backslash\{(0,0)\}$ parametrized by $\theta \in[0,2 \pi]$, for which the crossings of the negative $p_{1}$-axis in $\mathbb{R}^{2} \backslash\{(0,0)\}$ are in one-to-one correspondence with the decreasing zeros of $x \mapsto f(x)=q(x)+a x+b$ ( $a$ and $b$ fixed).

To that end, suppose $R \geq R_{0}$ is so large that $C(0, R)$ contains all the zeros of $q^{\prime \prime}$, which includes all the cusps of $\operatorname{cat}(q)$. Then the two components of $\operatorname{cat}(q)$ outside $C(0, R)$ are convex curves. It follows that each of them has at most one tangent line which passes through $(a, b)$. By making $R$ larger if necessary, we ensure that all the lines tangent to cat $(q)$ which pass through $(a, b)$ have their tangent points inside $C(0, R)$.

Let the two intersection points of $C(0, R)$ and $\operatorname{cat}(q)$ correspond to the parameter values $x_{0}<x_{1}$. We then have a curve $\mathbf{S}$ as described in $\S 2$. It lies in the nature of our problem that we construct $P$ by letting an $X Y$ frame move about a fixed point, namely $(a, b)=\left(p_{1}(\theta), p_{2}(\theta)\right)$; rather than letting a point move in $\mathbb{R}^{2}$ with respect to a fixed $X Y$ frame. In fact, we let the origin of an orthonormal $X Y$ frame slide over $\mathbf{S}$ in such a manner that, except at the points $x_{0}$ and $x_{1}$, the $X$-axis is tangent to $\mathbf{S}$. This makes for a parametrization of the form

$$
\begin{aligned}
& p_{1}(\theta)=(a-\alpha(\theta)) \cos \phi(\theta)-(b-\beta(\theta)) \sin \phi(\theta), \\
& p_{2}(\theta)=(a-\alpha(\theta)) \sin \phi(\theta)+(b-\beta(\theta)) \cos \phi(\theta),
\end{aligned}
$$




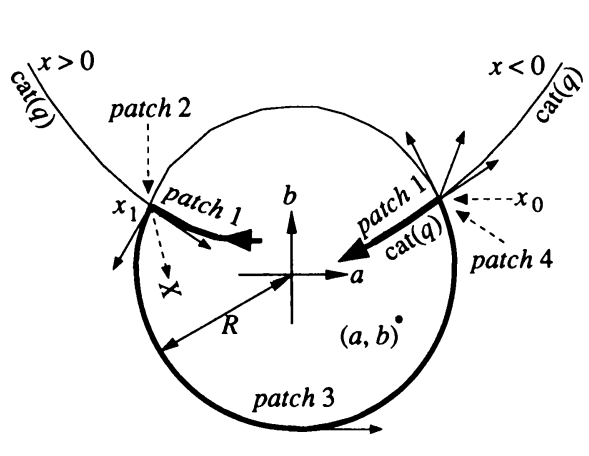

(even case)

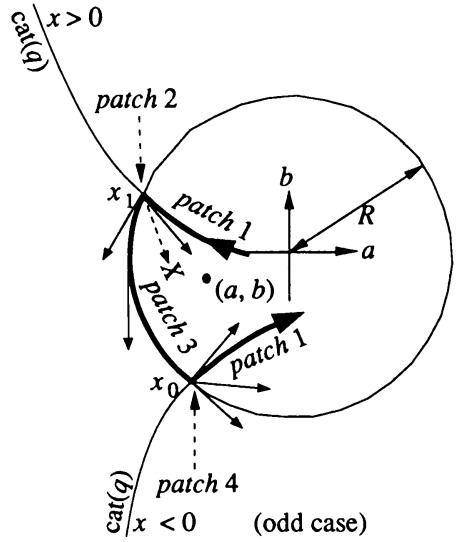

FIGURE 1

where the parameters $\phi(\theta), \alpha(\theta)$ and $\beta(\theta)$ are yet to be suitably defined. Since $\mathbf{S}$ consists of four patches (see Figure 1), we shall describe how the positive $X$ axis will change direction on each of the four patches in terms of these three parameters (patches 2 and 4 are the points $x_{1}$ and $x_{0}$ respectively, where the $X$-axis rotates only). We offer two descriptions of these orientations, viz. an informal "kinematical" one and a formal one. The reader could skip any of the two if he so wishes.

Kinematical description of the patches:

- patch 1. For $\theta \in\left[\operatorname{Arctg} x_{0}, \operatorname{Arctg} x_{1}\right]$, the positive $X$-axis is the half line $\widetilde{S}_{x}$ that is tangent at $x=\operatorname{tg} \theta$ to $\operatorname{cat}(q)$, oriented towards positive $a$. Its starting point is $(A(\operatorname{tg} \theta), B(\operatorname{tg} \theta))$ on $\operatorname{cat}(q)$.

- patch 2. For $\theta \in\left[\operatorname{Arctg} x_{1}, \pi / 2\right]$, the origin of the $X$-axis is fixed on $\operatorname{cat}(q)$ at $x=x_{1}$; this is a point in the second quadrant of the $(a, b)$ plane. The direction of the $X$-axis moves clockwise, starting from the tangent to $\operatorname{cat}(q)$ and ending at the anticlockwise tangent to $C(0, R)$.

- patch 3. For $\theta \in[\pi / 2,3 \pi / 2]$, the origin of the $X$-axis is a point on $C(0, R)$ moving anticlockwise from $\operatorname{cat}(q)\left(x_{1}\right)$ to cat $(q)\left(x_{0}\right)$. The direction of the positive $X$-axis is the anticlockwise tangent to $C(0, R)$.

- patch 4. For $\theta \in\left[3 \pi / 2, \operatorname{Arctg} x_{0}\right]$, the origin of the $X$-axis is fixed on $\operatorname{cat}(q)$ at $x=x_{0}$; this is in the odd case (resp. even case) a point in the third (resp. first) quadrant of the $(a, b)$-plane. The direction of the positive $X$-axis moves, starting from the anticlockwise tangent to $C(0, R)$, in anticlockwise (resp. clockwise) fashion and, in both cases, ends up at the "positive $a$ " tangent to $\operatorname{cat}(q)$.

Formal description of the patches:

In order to give the explicit values of the parameters $\phi(\theta), \alpha(\theta)$ and $\beta(\theta)$ for the different patches, we introduce six auxiliary angles and agree for convenience that in the $(a, b)$-plane their clockwise fashion is counted positive. 
They are given by the formulas

$$
\begin{gathered}
\operatorname{tg} \phi_{0}=x_{0}, \quad-\pi / 2<\phi_{0}<0, \\
\operatorname{tg} \phi_{1}=x_{1}, \quad 0<\phi_{1}<\pi / 2, \\
R \cos \psi_{0}=A\left(x_{0}\right), \quad R \cos \psi_{1}=A\left(x_{1}\right), \quad\left\{\begin{array}{l}
-\pi<\psi_{0}<-\pi / 2 \quad \text { (odd), } 0<\psi_{0}<\pi / 2 \quad \text { (even), } \\
\pi / 2<\psi_{1}<\pi .
\end{array}\right. \\
R \sin \psi_{0}=B\left(x_{0}\right), \quad R \sin \psi_{1}=B\left(x_{1}\right), \quad\left\{\begin{array}{l}
0<\phi_{0}^{\prime}<\pi / 2 \text { (odd), } \\
-\pi<\phi_{0}^{\prime}<-\pi / 2 \text { (even), } \\
\pi / 2<\phi_{1}^{\prime}<\pi .
\end{array}\right. \\
\phi_{0}^{\prime}=-\pi / 2-\psi_{0}, \quad \text { ) }
\end{gathered}
$$

For the different patches the values are as follows:

- patch 1. $\left(\phi_{0} \leq \theta \leq \phi_{1}\right)$

$$
\phi(\theta)=\theta, \quad \alpha(\theta)=A(\operatorname{tg} \theta), \quad \beta(\theta)=B(\operatorname{tg} \theta) .
$$

- patch 2. $\left(\phi_{1} \leq \theta \leq \pi / 2\right)$

$$
\begin{aligned}
& \phi(\theta)=\phi_{1}+\frac{\theta-\phi_{1}}{\pi / 2-\phi_{1}}\left(\phi_{1}^{\prime}-\phi_{1}\right), \\
& \alpha(\theta)=A\left(x_{1}\right), \quad \beta(\theta)=B\left(x_{1}\right) .
\end{aligned}
$$

- patch 3. $(\pi / 2 \leq \theta \leq 3 \pi / 2)$

$$
\begin{aligned}
& \phi(\theta)=\phi_{1}^{\prime}+\frac{\theta-\pi / 2}{\pi}\left(\phi_{0}^{\prime}-\phi_{1}^{\prime}\right), \\
& \alpha(\theta)=R \cos ((3 \pi / 2-\phi(\theta)), \quad \beta(\theta)=R \sin ((3 \pi / 2-\phi(\theta)) .
\end{aligned}
$$

- patch $4 .\left(-\pi / 2 \leq \theta \leq \phi_{0}\right)$

$$
\begin{aligned}
& \phi(\theta)=\phi_{0}^{\prime}+\frac{\theta+\pi / 2}{\phi_{0}+\pi / 2}\left(\phi_{0}-\phi_{0}^{\prime}\right), \\
& \alpha(\theta)=A\left(x_{0}\right), \quad \beta(\theta)=B\left(x_{0}\right) .
\end{aligned}
$$

Note that both the origin of the $X$-axis and its direction vary continuously as $\theta$ runs through the unit circle $[0,2 \pi]$. We claim that the direction of the $X$-axis has zero winding in the $(a, b)$-plane. In fact, it does not even cover the unit circle; e.g. it never has the direction of the negative $a$-axis. This implies that the winding number of the origin of the $X$-axis around $(a, b)$ is equal to the winding number of $\mathbf{S}$ around $(0,0)$ in the $\left(p_{1}, p_{2}\right)$-plane.

We now prove that this number is also equal to the number of decreasing zeros of $f$. First note that by the choice of $R$, the negative $X$-axis never contains the point $(a, b)$ in patches two, three and four (for the even case in patch four, look at the construction of $R$ ). It follows that $\mathbf{S}$ does not cross the negative $X$-axis for $\theta \in\left[\operatorname{Arctg} x_{1}, \operatorname{Arctg} x_{0}\right]$. Next, for $\theta \in\left[\operatorname{Arctg} x_{0}, \operatorname{Arctg} x_{1}\right]$, the positive $X$-axis equals $\widetilde{S}_{x}$, so that (cf. Equation (1)) crossings of the negative $p_{1}$-axis correspond to $\operatorname{tg} \theta$ being a decreasing zero of $f$, while crossings of the positive $p_{1}$-axis correspond to $\operatorname{tg} \theta$ being an increasing zero.

Hence, for $\theta \in\left[\operatorname{Arctg} x_{0}, \operatorname{Arctg} x_{1}\right]$, crossings of the $p_{1}$-axis are alternating on its positive and negative halves, i.e., they correspond to increasing and 
decreasing zeros, respectively. Therefore the number of each is the winding number of $\mathbf{S}$ indeed. This establishes the theorem.

\section{REFERENCES}

1. W. Kuyk and L. Smits, On the geometries of the rational unfoldings of $X^{k}$, Acta Appl. Math. (to appear).

University of ANTWerp (UIA), Departement Wiskunde, Universiteitsplein 1, B-2610 WILRIJK, BELGIUM

University of ANTWerp (RUCA), Dienst Algebra, GroenenborgerlaAn 171, B-2020 ANTWERP, BELGIUM 